\title{
PDE-5 inhibitors in monotherapy versus combination therapy in a sample of 1200 patients with erectile dysfunction
}

\author{
Luis Labairu-Huerta ${ }^{1}$, Bárbara Padilla-Fernández ${ }^{2}$, José Luis Arrondo-Arrondo ${ }^{3}$, Lauro Sebastián \\ Valverde-Martínez ${ }^{4}$, Agustín Martín-Rodríguez ${ }^{4}$, Juan Miguel Silva-Abuín ${ }^{5}$, María Begoña García- \\ Cenador ${ }^{6}$, José Antonio Mirón-Canelo ${ }^{7}$, María Fernanda Lorenzo-Gómez 4, 6, 8 \\ ${ }^{1}$ Department of Urology, University Hospital of Donostia, Spain; \\ ${ }^{2}$ Department of Urology, University Hospital of the Canary Islands, Tenerife, Spain; \\ ${ }^{3}$ Department of Urology, Hospital of Navarra, Spain; \\ ${ }^{4}$ Department of Urology, University Hospital of Salamanca, Spain; \\ ${ }^{5}$ Department of Urology, University Hospital of San Pedro, Logroño, Spain; \\ ${ }^{6}$ Department of Surgery, University of Salamanca, Spain; \\ ${ }^{7}$ Department of Preventive Medicine and Public Health, University of Salamanca, Spain; \\ ${ }^{8}$ Urological Renal Multidisciplinary Research Group of the Biomedical Research Institute of Salamanca (IBSAL), Spain.
}

\begin{abstract}
Summary Objectives: To compare the effectiveness in the treatment of erectile dysfunction when using PDE-5 inhibitors (PDE5i), alprostadil (PG-E1) and testosterone (TES) in monotherapy or combination therapy. Material and Methods: Observational multicentre retrospective study of men diagnosed and treated for ED between January 2008 and January 2014. Age, social and employment situation, pathological medical history, risk factors, usual treatments, IIEF-5 at the first consultation and at first and each 6 months follow-ups, physical examination, calculated total and free testosterone and received treatment were analysed. Descriptive statistics, one-way ANOVA analysis, Chi2 for qualitative data, t-test, Fisher's exact test and Pearson's correlation coefficient were used; $p<0.05$ is considered significant.

Results: Average age was 58.61 years, SD5.02, average follow-up time 48.21 months, SD 6.21, range 6-174 months. Out of the patients $76.12 \%$ were married, $9.81 \%$ divorced/separated, $10.04 \%$ single, $4.03 \%$ widowed; $85.14 \%$ of the total in stable partnership but $66.16 \%$ were not accompanied by their partners. In total 844 patients received monotherapy (597 PDE5i; 62 PG-E1; 36 TES; 27 penile prosthesis; 121 psychotherapy/alternative therapies) and 357 combination therapy (167 PDE5i+TES; 124 PDE5i+PGE1; 66 PG-E1+TES). There was a homogeneous distribution between risk factors and medical history groups. Satisfactory response according to IIEF-5 was achieved for $72.33 \%$ of patients on PDE5i monotherapy, $46.65 \%$ of patients on PDE5i+PG-E1 combination therapy and $83.41 \%$ of patients on PDE5i+TES.

Conclusions: The best therapeutic success for ED in this series was achieved through a combination of testosterone+PDE-5 inhibitors without increasing morbidity and maintaining the response over time. Larger studies with longer follow-up will corroborate these findings.
\end{abstract}

KEY WORDS: Erectile dysfunction; Combination therapy; PDE-5 inhibitors; Testosterone.

Submitted 8 January 2015; Accepted 31 March 2015

\section{INTRODUCTION}

Erectile dysfunction (ED) is defined as the persistent and/or recurrent inability to achieve and maintain sufficient rigidity of penis to allow satisfactory sexual performance (1), extended for at least three months, except for the cases secondary to trauma or surgery, or those cases problematically experienced by the patient (2).

The World Health Organization expressly recognizes that sexual health is a basic right of the individual and that erectile dysfunction is a serious disease that changes the quality of life of those suffering it, their partners and families, and that can be framed in the group III $(3,4)$.

ED is currently considered a health indicator, i.e. an alarm signal that can predict the presence of serious cardiovascular diseases (5).

However, most cases have a multifactorial origin and for almost all men, ED will be accompanied by some psychological disorder that is sometimes the only cause of the dysfunction perpetuation (6). The main objective of the treatment strategy for ED patients is to determine the aetiology of the disease, to treat it whenever possible and not to treat only the symptoms.

On the $1^{\text {st }}$ International Consultation on ED, it was established that modifiable risk factors should be corrected for initial treatment, and that therapeutic proposals staggered from the least to the most aggressive and individualised for each patient should be provided (3).

The first-line drug treatment is established with phosphodiesterase-5 inhibitors; testosterone should be used for patients with proved hypogonadism, and prostaglandin-El for those who don't respond or cannot take PDE5i (7).

However, we postulate that monotherapy with PDE-5 inhibitors (PDE5i), prostaglandin E1 (PG-E1) and testosterone (TES) may be less effective than combination therapy with 2 or 3 components. 


\section{Materials AND METHOdS}

A retrospective observational multicentre study was designed in order to assess the effectiveness of different therapeutic modalities for ED.

Medical records of men diagnosed and treated for ED between January 2008 and January 2014 at the Andrology consultations of the University Hospital of Salamanca and the Hospital of Navarra were reviewed. Age, social and employment situation, pathological medical history, risk factors, usual treatments, IIEF and IIEF5 questionnaire score at the first consultation and at first month and each 6 months follow-ups, physical examination, calculated total and free testosterone and received treatment were collected.

A descriptive study of the distribution of the frequency of every studied qualitative variable, and the corresponding descriptive statistics (central tendency and dispersion measures) of the quantitative variables included in the study (univariate analysis) was carried out for the statistical treatment of the information. A variables association study (bivariate analysis) was then performed in order to assess the relationship, statistically significant, $\mathrm{p}$ $<0.05$, among the variables included in the study. Parametric and nonparametric tests based on the characteristics of the variables and the adequate procedures and tests have been used in this process.

The response to the 5 questions IIEF questionnaire was classified as follows:

No response (0): score between 5 and 16;

Partial/slight response (1): score between 17 and 21;

Satisfactory response (2): score between 22 and 25.

\section{RESULTS}

During the study period, 1200 men diagnosed and treated for ED were identified in Salamanca and Navarra (Spain).

The average age was 58.61, ranging between 43 and 75 . The average follow-up time was 48.21 months, SD 6.21 with a minimum follow-up time of 6 months and a maximum of 174 months. Out of all the patients $76.12 \%$ were married, 9.81\% divorced or separated, $10.04 \%$ single and $4.03 \%$ widowed (Figure 1).

\section{Figure 1.}

Distribution of married, divorced or separated, single and widowed patients.

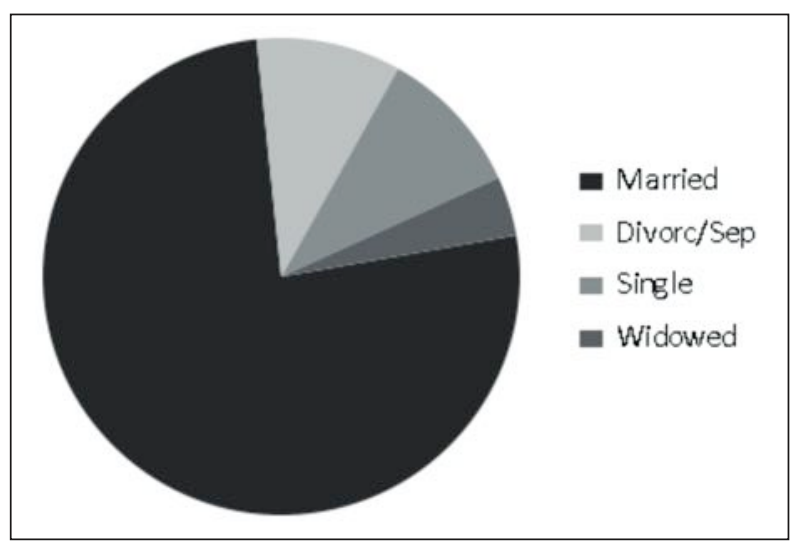

Table 1.

Distribution of patients included in each group.

\begin{tabular}{|l|l|c|}
\hline & Treatment received & Number of patients \\
\hline Monotherapy & PDE5i Vardenafil 20 mg & 272 \\
& Sildenafil 25-50-100 mg & 171 \\
& Tadalafil 10-20 mg & 154 \\
& Alprostadil & 62 \\
& Testosterone & 36 \\
& Penile prosthesis & 27 \\
& Others & 121 \\
\hline Combination & PDE5i+TES & 167 \\
therapy & PDE5i+PG-E1 & 124 \\
& PG-E1+TES & 66 \\
\hline
\end{tabular}

In general $85.14 \%$ of the total reported having a stable partner, but $66.16 \%$ were not accompanied by their partners. Furthermore, $61.83 \%$ reported having normal ejaculations and $35.02 \%$ reported maintaining normal sexual desire.

In total 844 patients were in treatment with monotherapy and 356 with combination therapy. Eight groups were differentiated according to the drug received:

Group A: phosphodiesterase inhibitors 5; Group B: alprostadil; Group C: testosterone; Group D: penile prosthesis; Group E: other treatments (psychotherapy, alternative therapies); Group F: PDE5i and testosterone combination; Group G: PDE5i and alprostadil combination; Group H: alprostadil and testosterone combination. Table 1 shows the number of patients included in each group.

Table 2 shows a summary of the patients' response to the IIEF-5 questionnaire in the different study groups. At the first visit, all patients have an unsatisfactory erectile function; then the percentage of intra-group patients regarding the satisfaction degree at first month and each 6 months follow-ups after establishing the different therapeutic measures is shown in percentage.

Regarding the medical history of men, $42.01 \%$ suffered from hypertension; 20.03\% from diabetes mellitus; $30.42 \%$ from obesity (BMI > 30); $11.30 \%$ from some type of heart disease and $10.32 \%$ from psychiatric disorders. Furthermore $64.33 \%$ were active smokers (5-40 cigarettes /day) and $69.39 \%$ reported alcohol consumption of $20 \mathrm{~g}$ or higher. The patients were on polypharmacy in $70.01 \%$ : $46.38 \%$ was taking anti-hypertensive drugs, $12.24 \%$ 5o-reductase inhibitors and $10.09 \%$ antidepressant. Finally $6.52 \%$ of patients suffered from prostate cancer and $3.02 \%$ had undergone radical prostatectomy. No statistically significant differences between groups regarding these records were proved. Satisfactory response was found for $72.33 \%$ of patients under PDE5i monotherapy, $46.65 \%$ of patients under combination therapy with PDE5i+ PG-El and $83.41 \%$ of patients under PDE5i+TES. The mortality in the series was $2.01 \%$, although these deaths were not related to ED. In fact there was no relation between death and erectile dysfunction or received treatment in any case.

Administration of TES was stopped in two cases due to 
Table 2

Summary of the patients' response to the IIEF-5 questionnaire in the different study groups.

\begin{tabular}{|c|c|c|c|c|c|c|c|c|c|c|c|c|c|c|c|c|c|c|c|}
\hline \multirow[b]{2}{*}{ Response to IIEF-5 } & \multirow{2}{*}{$\frac{\mathbf{1}^{\text {st }} \mathbf{V}}{0(\%)}$} & \multicolumn{3}{|c|}{ F/U 1st month } & \multicolumn{3}{|c|}{ F/U 12 months } & \multicolumn{3}{|c|}{ F/U 24 months } & \multicolumn{3}{|c|}{ F/U 36 months } & \multicolumn{3}{|c|}{ F/U 48 months } & \multicolumn{3}{|c|}{ F/U 72 months } \\
\hline & & $0(\%)$ & $1(\%)$ & $2(\%)$ & $0(\%)$ & $1(\%)$ & $2(\%)$ & $0(\%)$ & $1(\%)$ & $2(\%)$ & $0(\%)$ & $1(\%)$ & $2(\%)$ & $0(\%)$ & $1(\%)$ & $2(\%)$ & $0(\%)$ & $1(\%)$ & $2(\%)$ \\
\hline Group A & 100 & 12 & 14 & 74 & 13 & 15 & 72 & 14 & 15 & 71 & 15 & 15 & 70 & 15 & 16 & 69 & 14 & 15 & 66 \\
\hline Group B & 100 & 5 & 30 & 65 & 6 & 31 & 63 & 7 & 30 & 63 & 6 & 31 & 63 & 7 & 32 & 61 & 8 & 30 & 60 \\
\hline Group C & 100 & 47 & 6 & 47 & 42 & 14 & 44 & 40 & 16 & 44 & 38 & 18 & 44 & 36 & 18 & 46 & 33 & 19 & 47 \\
\hline Group D & 100 & 19 & 24 & 57 & 18 & 25 & 57 & 19 & 25 & 56 & 18 & 26 & 56 & 17 & 26 & 57 & 15 & 24 & 56 \\
\hline Group E & 100 & 54 & 18 & 28 & 50 & 20 & 30 & 51 & 21 & 28 & 52 & 22 & 26 & 55 & 23 & 22 & 53 & 21 & 20 \\
\hline Group F & 100 & 6 & 11 & 83 & 6 & 10 & 84 & 5 & 11 & 84 & 5 & 12 & 83 & 5 & 11 & 84 & 6 & 10 & 82 \\
\hline Group G & 100 & 20 & 36 & 44 & 19 & 37 & 44 & 18 & 38 & 44 & 19 & 36 & 45 & 20 & 36 & 44 & 21 & 33 & 40 \\
\hline Group H & 100 & 13 & 50 & 37 & 12 & 50 & 38 & 11 & 51 & 38 & 12 & 50 & 38 & 10 & 49 & 41 & 9 & 45 & 42 \\
\hline
\end{tabular}

the increase in packed cell volume, in one case due to PSA increase with no confirmation of prostate cancer after biopsy and in two other cases due to mood changes. The overall response to the treatment was satisfactory in $70.04 \%$, partial in $16.09 \%$ and there was no response in $13.87 \%$. No changes were seen in the annual follow-ups $(\mathrm{p}=0.4836)$ after analysing the responses to the IIEF questionnaire.

\section{Discussion}

A number of studies have shown the importance of sexual health and its direct influence on psychological wellbeing of individuals and their partners (4). Society evolution is oriented towards a even greater importance of sexual health in the future (8). However, it is estimated that only $16.5 \%$ of patients with erectile dysfunction go to the doctor in order to be diagnosed and treated, mainly because both doctors and patients are still reluctant to treat sexual issues openly at the consultation.

It is estimated that over 320 million men will suffer from ED in Western countries, which means more than twice the observed prevalence 15 years ago. This invites us to reassess the therapeutic strategy of this pathology $(9,10)$. The main objective of the treatment strategy for patients with ED is to determine the aetiology of the disease, to treat it whenever possible and not to treat only the symptoms. It is very important to encourage changes in the patient's lifestyle in order to decrease the incidence of cardiovascular diseases (11): doing regular exercise, stop smoking, healthy diet, drinking little alcohol. The potential benefits of lifestyle changes may be particularly important in patients with ED and selected concomitant cardiovascular or metabolic diseases such as diabetes or hypertension $(12,13)$. In fact, studies show that intensive changes in lifestyle not only improve erectile function, but can be also beneficial for overall cardiovascular and metabolic health (14). Once risk factors are identified and corrected, the first-line drug treatment is established with phosphodiesterase-5 inhibitors in monotherapy, with a broad safety profile even in patients with multiple pathologies, considering combination therapy as second-line (15). The main reason why a patient does not use properly PDE5i is the inadequate advice of the physician, who may encourage not using adequate sexual stimulation, not using the appropriate dose or not waiting enough time between the drug intake and the attempt of intercourse (16). A drug must be taken at least 6 times in order to test its effectiveness (17) and it must be taken at maximum dose. Furthermore, the right patient education has been found to help achieving the effective response of a PDE5 inhibitor without apparent previous response after underlining the importance of dose, the moment of intake and the necessary sexual stimulation $(18,19)$. A second-line setting was performed in one study and patients treated with tadalafil were advised to wait at least two hours; the patients treated with vardenafil were advised to use it fasting, rescuing patients with no apparent response (20). Chronic therapy with PDE5i is safe and is an alternative in the treatment of ED in any degree or aetiology, although no subpopulation of men with ED taking. greater advantages from chronic therapy has been identified, but it is promoted as the best alternative for men who seek for more naturalness and spontaneity. Furthermore, chronic inhibition of PDE-5 might be a treatment of endothelial dysfunction. Tadalafil $5 \mathrm{mg}$ is the only drug approved in this sense (21). Two non-randomized trials have showed that daily intake of a PDE5i can rescue some patients who do not respond to intermittent administration: in one study, some patients benefited from regular administration of vardenafil or tadalafil (22); in other study, daily administration of tadalafil rescued patients who had not responded to intermittent administration of a PDE-5 inhibitor (23). Tadalafil is the only drug approved for daily administration of a dose of 2.5 to 5 mgr.

Sildenafil, tadalafil and vardenafil can be nowadays considered equally effective and safe, therefore it is postulated that patients should have the opportunity of trying the three drugs and then select the one that best suits their sexual habits and is most effective (24). Some authors have studied the pharmacological combination value in ED, especially in those cases refractory to conventional treatment $(10,25)$. In patients with testosterone deficiency, normalization of serum testosterone may improve the response to PDE5i: Shabsigh et al. (26) studied the administration of testosterone gel and sildenafil 100 mgr versus sildenafil 100mgr in a double-blind 
study with 75 hypogonadal men with ED for 12 weeks. Combination therapy group patients significantly improved the IIEF-EF domain (4.4 vs. 2.1) and better but not significant scores were observed in the IIEF-Q3Q4. One patient left the combination therapy due to adverse event. Aversa et al. (27) assessed administration of patches of testosterone $5 \mathrm{mgr}$ and sildenafil $100 \mathrm{mgr}$ versus sildenafil $100 \mathrm{mgr}$ in monotherapy. In this nonmasking study, 77 hypogonadal men with ED were studied for one month. The group under combination therapy had a significant improvement of the IIEF (21.8 vs. 14.2), of the number of intercourses (2.8 vs. 1.5), of satisfactory intercourses (12.1 vs. 7.7) and of erections ( $80 \%$ vs. $10 \%)$. The testosterone replacement therapy can be provided with gel at $1 \%$ achieving improvement from the first month in the IIEF, although with no statistical significance (26). The hypogonadal men can receive TRT and add tadalafil with good response or receive vardenafil when they do not respond to testosterone undecanoate monotherapy, with good response in $88 \%$ and better satisfaction of their partners (26). In another case series published by Greenstein et al. (28), it was observed that in 17 out of 49 hypogonadal patients in whom TES monotherapy had failed, a significant improvement of erectile function according to the IIEF questionnaire was achieved after combination with sildenafil. In addition, all the 49 patients reported to be satisfied with their erections under combination therapy.

Prevalence of total testosterone deficiency in men with ED is highly variable depending on the assessed studies, showing a broad range from $1.72 \%$ to $47.7 \%$, although studies with largest number of patients set it at 5.7\% (29), $6.65 \%$ (30) or $15 \%$ (31). A significant association between the decrease of free testosterone and insulin resistance has also been proved, which could justify the use of TES as combination therapy in diabetic patients without prostate cancer (32). TES replacement therapy is contraindicated in patients with history of prostate cancer or with prostatism symptoms. Rectal examination and a PSA test should be performed before starting this treatment. Clinical response and emergence of liver disease or prostate condition (33) should be then controlled. There are no contraindications in the use of TES in men with coronary disease correctly diagnosed as hypogonadism or ED, although packed cell volume should be monitored and testosterone dose adjustment may be necessary, especially in patients with congestive heart failure. The PG-El, marketed as alprostadil, is considered the second-line treatment, mainly administered as intracavernous injections at 5-40 mg doses (11). The right technique for the drug administration should be taught to the patient, which is also useful in order to verify successful response to the treatment. Effectiveness' rates are around $70 \%$; sexual activity is presented after $94 \%$ of injections, with satisfaction rates ranging from 87 to $93.5 \%$ in patients and from 86 to $90.3 \%$ in their partners (11). Intracavernous therapy is however accompanied by high dropout rates ranging from 41 to $68 \%$ and its compliance is limited (34). The first combination of intracavernous therapy was performed by Zorgniotti in 1985 with papaverine and phentolamine (35). It is still indicated in patients with PG-El painful injections or when PG-El monotherapy becomes ineffective (36). A physiological study proves that this combination therapy of ICI with Trimix and sildenafil has better results in Doppler ultrasound and higher increase of guanosine monophosphate (cGMP) and adenosine monophosphate (cAMP) in the corpus cavernosum (37). Only two studies were published about this combination therapy in patients who did not report problems with priapism and referred difficulty in adjusting doses: the first study included 93 patients who did not responded to ICI (Trimix), of which 34\% responded to sildenafil as rescue therapy; $48 \%$ of the rest had a good response to combination therapy, but had more side effects (38). The second study with 22 post-RP patients, in which better results were obtained by combination therapy (sildenafil + Trimix) versus monotherapies (39). The use of sildenafil and alprostadil has been successful after failure of monotherapy with sildenafil, although success rates vary from 47 to $100 \%$ (25). This therapy has been used even in patients who had undergone retro-pubic radical prostatectomy with nerve preservation, with satisfactory results $(39,40)$. The key to the success of this association is the use of two different cavernous ways: PDE5 inhibitors indirectly relax body's smooth muscle by inhibiting the metabolism of cyclic guanosine monophosphate, for which nitric oxide is required; PGEl directly relaxes trabecular smooth muscle tissue through E-prostanoid receptors, which leads to an increase of cyclic adenosine monophosphate. Therefore, the use in combination of these two drugs theoretically allows a therapeutic synergy to activate two different but inter-related ways $(10,41)$. In our series, patients did not report suffering severe adverse effects with this combination, and in particular no higher incidence of priapism compared to monotherapy group. There is no scientific evidence of previous use of PG-El and TES in combination therapy (10). In one study with 120 men who had not responded to sildenafil or intraurethral alprostadil monotherapy, it is assessed what happens when both are combined. None stopped the combination therapy due to adverse effects and they improved the IIEF score (42). Other studies have reported the same with smaller series of patients (43), even in patients who had undergone radical prostatectomy who had not responded to monotherapy and in both studies with no increase of adverse effects after combination therapy (44). A metaanalysis with 515 men with ED and LUTS concluded that combination therapy is more effective than PDE5i, with better scores on the IIEF questionnaires, IPSS, and peak flow (45).

A meta-analysis with 398 men showed better effectiveness and greater adherence to treatment when psychosexual therapy is combined with PDE5i in comparison to simple administration of PDE5i $(46,47)$.

\section{Conclusions}

The best therapeutic success for ED in our series is achieved by combining testosterone and PDE-5 inhibitors without increasing morbidity and keeping the response over time. Waiting for the results of monotherapy instead of establishing a combination therapy initial- 
ly leads to lower success of therapy as well as to stop trying and a more negative attitude. It also increased anxiety of patients who did not have a satisfactory answer. We advise using combination drug therapies as the effects are well known and different in order to promote synergy of treatment by helping to solve the various mechanisms involved in erectile dysfunction.

Larger studies with longer follow-up will corroborate these findings.

\section{REFERENCES}

1. Resel Estévez L, Silmi Moyano A, Moreno Sierra J. Disfunción Eréctil. 2004.

2. Ralph D, McNicholas T. UK management guidelines for erectile dysfunction. BMJ. 2000;32:499-503.

3. Erectile Dysfunction. World Health Organization, editor. Oxford. United Kingdom.: Health Publication Ltd. Plymouth.; 2000.

4. Feldman H, Goldstein I, Hatzichristou D, et al. Impotence and its medical and psychosocial correlates: results of the Massachusetts Male Aging Study. J Urol. 1994; 151:54-6.

5. Burchardt M, Burchardt T, Anastasiadis A, et al. Erectile dysfuncion is a marker for cardiovascular complications and psychological functioning in men with hypertension. Int J Impot Res. 2001; 13:276-81.

6. Martín Morales A, Díaz F, García E. Epidemología y etiopatogenia de la disfunción eréctil. In: Rodríguez Vela L, Rioja Sanz L, editors. Actualización en Andrología. Barcelona: Ediciones Pulso; 2000.

7. Eardley I, Donatucci C, Corbin J, et al. Pharmacotherapy for Erectile Dysfunction. J Sex Med 2010; 7 (1pt2):524-40.

8. Fugl-Meyer A, Lodnert G, Bräholm I, Fugl-Meyer K. On life satisfaction in male erectile dysfunction. Int J Impot Res. 1997; 9:141-8.

9. McKinlay J. The worldwide prevalence and epidemiology of respective PDEs, could achieve greater enhance- erectile dysfunction. Int J Impot Res. 2000; 2(Suppl 4):S6-11.

10. Dhir RR, Lin H-C, Canfield SE, Wang R. Combination therapy for erectile dysfunction: an update review. Asian J Androl. 2011; 13:382-90.

11. Hatzimouratidis K, Amar E, Eardley I, et al. Guidelines on Male Sexual Dysfunction: Erectile Dysfunction and Premature Ejaculation. European Urology. 2010; 57:804-14.

12. Moyad M, Barada J, Lue T, et al. Sexual Medicine Society Nutraceutical Committee. Prevention and treatment of erectile dysfunction using lifestyle changes and dietary supplements: what works and what is worthless, part I. Urol Clin North Am. 2004; 31:249-57.

13. Moyad M, Barada J, Lue T, et al. Sexual Medicine Society Nutraceutical Committee. Prevention and treatment of erectile dysfunction using lifestyle changes and dietary supplements: what works and what is worthless, part II. Urol Clin North Am. 2004; 31:259-73.

14. Esposito K, Giugliano F, Di Palo C, et al. Effect of lifestyle changes on erectile dysfunction in obese men: a randomized controlled trial. JAMA. 2004; 291:2978-84.

15. Lue T. Erectile dysfunction. N Engl J Med. 2000; 342(Suppl. 24):1802-13.

16. Hatzimouratidis K, Amar E, Eardley I, et al. Guidelines on male sexual dysfunction: erectile dysfunction and premature ejaculation. Eur Urol 2010; 57:804-14.

17. Rajagopalan P, Mazzu A, Xia C, Dawkins R, Sundaresan P. Effect of high-fat breakfast and moderate-fat evening meal on the pharmacokinetics of vardenafil, an oral phosphodiesterase-5 inhibitor for the treatment of erectile dysfunction. J Clin Parmacol 2003; 43:260-7.

18. McCullough AR, Barada JH, Fawzy A, et al. Achieving treatment optimization with sildenafil citrate (Viagra) in patients with erectile dysfunction. Urology. 2002; 60 (2 Suppl 2):28-38.

19. Gruenwald I, Shenfeld O, Chen J, et al. Positive effect of counseling and dose adjustment in patients with erectile dysfunction who failed treatment with sildenafil. Eur Urol 2006; 50:134-40.

20. Hatzichristou D, Moysidis K, Apostolidis A, et al. Sildenafil failures may be due to inadequate patient instructions and follow-up: a study on 100 non-responders. Eur Urol 2005;47:518-22; discussion 22-3.

21. Fusco F, Razzoli E, Imbimbo C, et al. A new era in the treatment of erectile dysfunction: chronic phosphodiesterase type 5 inhibition. BJU international. 2010; 105:1634-9.

22. McMahon C. Efficacy and safety of daily tadalafil in men with erectile dysfunction previously unresponsive to on-demand tadalafil. J Sex Med 2004; 1:292-300.

23. Hatzimouratidis K, Moysidis K, Bekos A, et al. Treatment strategy for "non-responders" to tadalafil and vardenafil: a real-life study. Eur Urol 2006; 50:126-32; discussion 32-3.

24. Wespes E, Eardley I, Giuliano F, et al. Guidelines on Male Sexual Dysfunction: Erectile dysfunction and premature eyaculation. Arnheim, The Netherland, 2013.

25. Sommer F, Engelmann U. Future Options for Combination Therapy in the Management of Erectile Dysfunction in Older Men. Drugs \& Aging. 2004; 21:555-64.

26. Shabsigh R, Kaufman J, Steidle C, Padma-Nathan $H$. Randomized study of testosterone gel as adjunctive therapy to sildenafil in hypogonadal men with erectile dysfunction who do not respond to sildenafil alone. J Urol. 2004; 172:658-63.

27. Aversa A, Isidori A, Spera G, et al. Androgens improve cavernous vasodilation and response to sildenafil in patients with erectile dysfunction. Clin Endocrinol (Oxf). 2003; 58:632-8.

28. Greenstein A, Mabjeesh N, Sofer M, et al. Does sildenafil combined with testosterone gel improve erectile dysfunction in hypogonadal men in whom testosterone supplement therapy alone failed? Urology. 2005; 173:530-2.

29. Earle C, Stuckey B. Biochemical screening in the assessment of erectile dysfunction: what tests decide future therapy? Urology. 2003; 62:727-31.

30. Buvat J, Lemaire A. Endocrine screening in 1,022 men with erectile dysfunction: clinical significance and cost-effective strategy. J Urol. 1997; 158:1764-7.

31. El-Sakka A, Hassoba H, Sayed H, Tayeb K. Pattern of endocrinal changes in patients with sexual dysfunction. J Sex Med. $2005 ; 2: 551-8$

32. Guay A, Jacobson J. The relationship between testosterone levels, the metabolic syndrome (by two criteria), and insulin resistance in a population of men with organic erectile dysfunction. J Sex Med. 2007; 4:1046-55.

33. Morales A, Heaton J. Hormonal erectile dysfunction. Evaluation and management. Urol Clin North Am. 2001; 28:279-88.

34. Flynn RJ, Williams G. Long-term follow-up of patients with erectile dysfunction commenced on self injection with intracavernosal papaverine with or without phentolamine. Br J Urol. 1996; 78:628-31.

35. Zorgniotti AW, Lefleur RS. Auto-injection of the corpus cavernosum with a vasoactive drug combination for vasculogenic impotence. J Urol. 1985; 133:39-41. 
36. Vozmediano R. Tratamiento intracavernoso y dispositivos de vacío. In: Cruz N, editor. Tratado de andrología y medicina sexual 1. Sevilla: Editorial Médica Panamericana S.A.; 2011. p. 596-601.

37. Park JK, Park JS, Jeon SB, et al. Why a combined intracavernosal injection with trimix and oral sildenafil is reliable therapy in the ultrasonographic evaluation of erectile dysfunction. BJU international. 2008; 102:993-7.

38. McMahon CG, Samali R, Johnson H. Treatment of intracorporeal injection nonresponse with sildenafil alone or in combination with triple agent intracorporeal injection therapy. J Urol. 1999; 162:1992-7; discussion 7-8.

39. Nandipati K, Raina R, Agarwal A, Zippe CD. Early combination therapy: intracavernosal injections and sildenafil following radical prostatectomy increases sexual activity and the return of natural erections. Int J Impot Res 2006; 18:446-51.

40. Mydlo JH, Viterbo R, Crispen P. Use of combined intracorporal injection and a phosphodiesterase-5 inhibitor therapy for men with a suboptimal response to sildenafil and/or vardenafil monotherapy after radical retropubic prostatectomy. BJU International. 2005; 95:843-6.

41. Kim N, Huang Y, Moreland R, et al. Cross-regulation of intracellular cAMP and cGMP in cultured human corpus cavernosum smooth muscle cells. Mol Cell Biol Res Commun. 2000; 4:10-4.

42. Mydlo JH, Volpe MA, MacChia RJ. Results from different patient populations using combined therapy with alprostadil and sildenafil: predictors of satisfaction. BJU international. 2000; 86:469-73.

43. Nehra A, Blute ML, Barrett DM, Moreland RB. Rationale for combination therapy of intraurethral prostaglandin $E(1)$ and sildenafil in the salvage of erectile dysfunction patients desiring noninvasive therapy. Int J Impot Res 2002; 14 Suppl 1:S38-42.

44. Raina R, Nandipati KC, Agarwal A, et al. Combination therapy: medicated urethral system for erection enhances sexual satisfaction in sildenafil citrate failure following nerve-sparing radical prostatectomy. J Androl. 2005; 26:757-60.

45. Yan H, Zong H, Cui $Y$, et al. The efficacy of PDE5 inhibitors alone or in combination with alpha-blockers for the treatment of erectile dysfunction and lower urinary tract symptoms due to benign prostatic hyperplasia: a systematic review and meta-analysis. J Sex Med 2014; 11:1539-45.

46. Melnik T, Soares BG, Nasello AG. The effectiveness of psychological interventions for the treatment of erectile dysfunction: systematic review and meta-analysis, including comparisons to sildenafil treatment, intracavernosal injection, and vacuum devices. J Sex Med 2008; 5:2562-74

47. Schmidt HM, Munder T, Gerger H, et al. Combination of psychological intervention and phosphodiesterase-5 inhibitors for erectile dysfunction: a narrative review and meta-analysis. J Sex Med 2014; 11:1376-91.

\section{Correspondence}

Labairu-Huerta, Luis (Corresponding Author)

luislabairu@hotmail.com

Department of Urology, University Hospital of Donostia, Spain

Bárbara Padilla Fernández

padillaf83@hotmail.com

Department of Urology, University Hospital of the Canary Islands, Tenerife, Spain

Arrondo-Arrondo, José Luis

jlarrondo@telefonica.es

Department of Urology, Hospital of Navarra, Spain

Valverde-Martínez, Lauro Sebastián

sebasv_2000@hotmail.com

Martín-Rodríguez, Agustín

drmartinr@hotmail.com

Department of Urology, University Hospital of Salamanca, Spain

Silva-Abuín, Juan Miguel

elviso@usal.es

Department of Urology, University Hospital of San Pedro, Logroño, Spain

García-Cenador, María Begoña

mbgc@usal.es

Department of Surgery, University of Salamanca, Spain

Mirón-Canelo, José Antonio

miroxx@usal.es

Department of Preventive Medicine and Public Health. University of Salamanca, Spain

Lorenzo-Gómez, María Fernanda

mflorenzogo@yahoo.es

Department of Urology, University Hospital of Salamanca, Spain

Department of Surgery, University of Salamanca, Spain

Urological Renal Multidisciplinary Research Group of the Biomedical

Research Institute of Salamanca (IBSAL), Spain 